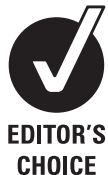

CHOICE

\title{
The effect of HIV infection on time off work in a large cohort of gold miners with known dates of seroconversion
}

\author{
Pam Sonnenberg, ${ }^{1}$ Andrew Copas, ${ }^{1}$ Judith R Glynn, ${ }^{2}$ Andre Bester, ${ }^{3}$ Gill Nelson, ${ }^{4,5}$ \\ Stuart Shearer, ${ }^{3}$ Jill Murray ${ }^{4,5}$
}

\begin{abstract}
${ }^{1}$ Research Department of Infection and Population Health, University College London, London, UK

${ }^{2}$ Department of Epidemiology and Population Health, London School of Hygiene and Tropical Medicine, London, UK

${ }^{3}$ Gold Fields Limited, Johannesburg, South Africa ${ }^{4}$ School of Public Health, University of the Witwatersrand, Johannesburg, South Africa

${ }^{5}$ National Institute for Occupational Health, National Health Laboratory Service, Johannesburg, South Africa
\end{abstract}

\section{Correspondence to}

Dr Pam Sonnenberg, Centre for Sexual Health \& HIV Research, Research Department of Infection and Population Health, Mortimer Market Centre, Capper Street, London WC1E 6JB, UK:

p.sonnenberg@ucl.ac.uk

Accepted 21 October 2010 Published Online First 28 November 2010

\section{ABSTRACT \\ Objectives To estimate the effect of HIV infection on time off work. To provide baseline estimates for economic and actuarial models, and for evaluations of ART and other workplace interventions.}

Methods A retrospective cohort study of gold miners with known dates of seroconversion to HIV, and an HIVnegative comparison group, used routinely collected data to estimate the proportion of time off work by calendar period (1992-2002, prior to the introduction of ART), age, time since seroconversion and period before death. The authors calculated ORs for overall time off work and $\mathrm{RR}$ ratios (RRR, using multinomial logistic regression) for reasons off work relative to being at work.

Results 1703 HIV-positive and 4859 HIV-negative men were followed for 34424 person-years. HIV-positive miners spent a higher proportion of time off work than negative miners $(20.7 \%$ vs $16.1 \%)$ due to greater medical and unauthorised absence. Compared with HIVnegative miners, overall time off work increased in the first 2 years after seroconversion (adjusted OR 1.40 (95\% Cl 1.36 to 1.45$)$ ) and then remained broadly stable for a number years, reaching $38.8 \%$ in the final year before death (adjusted OR 3.27, 95\% Cl 2.95 to 3.63). Absence for medical reasons showed the strongest link to HIV infection, increasing from an adjusted RRR of 2.66 (95\% Cl 2.45 to 2.90 ) for the first 2 years since seroconversion to $13.6(95 \% \mathrm{Cl} 11.8$ to 15.6$)$ in the year prior to death.

Conclusions Time off work provides a quantifiable measure of the effect of HIV on overall morbidity. HIV/ AIDS affects both labour supply (increased time off work) and demand for health services (increased medical absence). The effects occur soon after seroconversion and stabilise before reaching very high levels in the period prior to death. Occupational health services are an important setting to identify HIV-infected men early.

\section{INTRODUCTION}

The impact of the HIV/AIDS epidemic on workers, industry, economies and society is substantial, since HIV affects individuals in their productive working years of life. The consequences are most profound for labour-intensive industries (eg, mining and construction) which are also the sectors with the highest HIV prevalences in southern Africa. ${ }^{1}$ It is estimated that HIV prevalence on the South African gold mines was $1.3 \%$ in $1990,{ }^{2}$ rising to $20-30 \%$ by $1998 .^{3} 4$

Economic and actuarial models of the projected impacts of HIV/AIDS ${ }^{5-7}$ and the cost-effectiveness

\section{What this paper adds}

- Baseline data, prior to the introduction of antiretroviral therapy, are needed to assess and model the current and future impact of HIV in the working population and for the design and evaluation of appropriate interventions.

- Compared with HIV-negative miners, HIV-positive miners spent more time off work, mainly due to medical and unauthorised absence.

- The proportion of time off work increased soon after seroconversion and reached very high levels in the year before death.

- This paper quantifies the timing and effect of HIV/AIDS on labour supply (increased time off work) and demand for health services (increased medical absence).

- Occupational health services are an important setting in which to identify HIV-infected men early.

of interventions, including treatment, ${ }^{8}$ require robust estimates of model parameters. There is, however, a paucity of published work, based on empirical data, on the impact of HIV/AIDS on time off work. ${ }^{9-14}$ Existing studies are limited in that they are often based on relatively small numbers with short follow-up, lack an HIV-negative comparison group or use self-reported outcomes, and none have used seroconverter cohorts. Baseline data, prior to the introduction of antiretroviral therapy (ART), are needed to assess and model the current and future impact of HIV in the working population and for the design and evaluation of appropriate interventions.

This large cohort study of gold miners with known dates of seroconversion and an HIV-negative comparison group, estimates the effect of HIV/ AIDS and duration of infection on supply of labour (measured as time off work) and demand for health services (measured as time off work for medical reasons) prior to the rollout of ART.

The natural history of HIV has been extensively studied using clinical events, such as time to AIDS, or death as endpoints. ${ }^{15}$ We have previously estimated the effect of HIV on mortality, ${ }^{16}$ tuberculosis ${ }^{17}$ and work-related injuries. ${ }^{18}$ The impact of HIV on overall functioning and morbidity, especially in the early period following seroconversion, has not been described. This study therefore also provides a unique opportunity to estimate the 
impact of HIV/AIDS and how this changes by duration of infection using a quantifiable measure of morbidity.

\section{METHODS}

A retrospective cohort study was conducted among men working on four goldmines in South Africa, using routinely collected data. Free medical services are provided by the mine company, with ART introduced in 2004. Details of the study design have been published. ${ }^{16-18}$ Briefly, HIV tests were done with counselling and consent in two surveys in 1992-1993, in sexually transmitted disease clinics, and as clinically indicated, and results kept on a single confidential database. Individuals who were HIV-negative in one of the surveys and had no later evidence of HIV from tests or clinical diagnoses form the HIVnegative comparison group. The HIV seroconverter cohort is formed from individuals with an HIV-negative test and a subsequent HIV-positive test in either of the surveys or from testing done in clinics or the hospital (excluding tests done on medical or tuberculosis wards) until 1998. Consequently, the HIV-negative cohort was recruited in 1992-1993, with the seroconverters recruited in 1992-1998. We restricted the seroconverter cohort to individuals with intervals of less than 3 years between the last negative and first positive test. The seroconversion date was taken as the midpoint of the seroconversion interval. The study was approved by Ethics Committees at the University of the Witwatersrand, South Africa and LSHTM, UK.

The mine maintains a number of human resources system (HRS) databases, which include demographic information, employment history, payroll and time off work. The format of the routinely available datasets did not include time at work. We accessed an active database on currently employed men, as well as archived data on men who had left the mine. Records between 1 August 1992 and 1 October 2002 were available. In general, miners work 312 shifts per year, with up to 56 days leave (half of which is paid leave). In this context, 'at work' refers to doing routine activity (includes work, training, allocated rest days). For every employee, a record is entered for each episode off work, based on the dates of the first and last shifts missed. Reasons for being off work were grouped into medical, leave (annual, compassionate and unpaid leave) and unauthorised absence (including absence for an unknown reason). The HIV and HRS databases were linked using unique industry numbers. For construction of the cohort, HIV-negative men entered the analysis at the time of the negative test in the survey. HIV-positive men entered at the estimated date of seroconversion. Follow-up continued until the miner left the mine or died, or 1 October 2002.

We determined the proportion of time off work overall and by reason, with the overall number of days of follow-up as the denominator. We estimated the effect of HIV status on time off work compared with HIV-negative miners, with age, duration of HIV infection and calendar period as time-varying covariates. We calculated ORs using logistic regression for overall time off work. To investigate associations with each type of time off work, we assign time as either at work or in each of three types of time off. We then use multinomial logistic regression to simultaneously model the probabilities of time being spent in each of these four outcome categories. This regression generates $R R$ ratios (RRR) for each type of time off relative to being at work. For each predictor, the RRR is the ratio of the RR for the type of time off to the RR for being at work, which corresponds to the OR ignoring the other types of time off.
To determine the effect of end-stage HIV on time off work and reason, independent of time since seroconversion, we estimated the time off in the 2 years prior to death in those who died from natural causes, using data on date and cause of death collected as part of our larger study. ${ }^{16}{ }^{19}$ We compared HIV-positive men prior to death with HIV-negative men (overall and restricted to those who died).

Since days at work were not recorded, to assess completeness of the time off dataset, we identified gaps (no record of time off) of greater than 12 months and their relationship to study entry dates. In light of our findings (see Results), our primary analysis includes follow-up from the date of the first record in the time off database. To address a possible bias resulting from beginning follow-up at the first record of time off, we repeated the main analysis with subjects entering on the date of entry to the cohort. As a sensitivity analysis, we repeated the main analysis using the period before seroconversion as the baseline in the subgroup of miners with time off work records before and after seroconversion.

\section{RESULTS}

\section{Individuals included in the analysis}

A total of 1950 HIV-positive and 5814 HIV-negative miners were eligible for this study. Our initial analysis was based on the 1703 (87\%) HIV-positive and 4859 (84\%) HIV-negative men for whom records of time off work were available (table 1). Those miners with records available were younger, recruited later, more likely to be still employed in 2002 and more likely to be HIVpositive, and had longer study follow-up than those miners without records of time off work. When this comparison of miners included and excluded from our analysis was made separately for HIV-positive and -negative miners, the same differences were found, except that among HIV-negative miners there was no difference in the year of study recruitment (data not shown).

\section{Calculation of follow-up time}

The overall study follow-up included a total of 39781 person years. However, long gaps (no record of time off for $\geq 12$ months) make up 2538 person years in HIV-negative (8.1\% of total) and 1101 (13.1\%) in HIV-positive miners. These gaps are primarily at the start of study follow-up. Consequently, for our primary analyses, subjects entered follow-up at the date of the first documented period off work. This reduced the time represented by long gaps to only 296.0 person years $(1.1 \%)$ and 57.0 $(0.9 \%)$ in HIV-positive and HIV-negative men, respectively.

\section{Proportion of time off work by HIV status, calendar period, age and time since seroconversion}

Table 2 shows that, overall, $83.0 \%$ of follow-up time was at work, with miners being off work due to leave (13.1\% of time), for medical reasons $(2.7 \%)$ and unauthorised absence $(1.3 \%)$. In absolute terms, on average, HIV-negative miners are at work for 307 days per year and off sick for 8 days. In contrast, the respective numbers of days in HIV-positive miners are 290 and 20. Unauthorised absence declined with increasing age and over calendar period. HIV-positive miners spent more time off work than HIV-negative miners (20.7\% vs $16.1 \%$ ).

\section{Comparison of HIV-positive and HIV-negative miners who died} Among 361 HIV-positive miners who died from natural causes (table 3), $23.7 \%$ of time in the penultimate year was spent off work, rising to $38.8 \%$ in the final year, a high proportion of which was for medical reasons. The average number of days at 
Table 1 Comparison of miners by inclusion in the analysis and by HIV status

\begin{tabular}{|c|c|c|c|c|c|c|c|c|c|c|}
\hline \multirow[b]{3}{*}{ Factor } & \multicolumn{7}{|c|}{ Included in analysis } & \multirow{2}{*}{\multicolumn{2}{|c|}{$\begin{array}{l}\text { No records of } \\
\text { time off }\end{array}$}} & \multirow[b]{3}{*}{ p Value } \\
\hline & \multicolumn{2}{|c|}{ HIV-positive } & \multicolumn{2}{|c|}{ HIV-negative } & \multirow[b]{2}{*}{ p Value* } & \multicolumn{2}{|l|}{ Total } & & & \\
\hline & $\mathbf{n}$ & $\%$ & $\mathbf{n}$ & $\%$ & & $\mathbf{n}$ & $\%$ & $\mathbf{n}$ & $\%$ & \\
\hline Total & \multicolumn{2}{|l|}{1703} & \multicolumn{2}{|l|}{4859} & & \multicolumn{2}{|l|}{6562} & \multicolumn{2}{|c|}{1202} & \\
\hline \multicolumn{5}{|l|}{ Age (years) at start of study } & $<0.001$ & & & & & \multirow[t]{5}{*}{$<0.001$} \\
\hline $15-24$ & 264 & 15.5 & 555 & 11.4 & & 819 & 12.5 & 117 & 9.7 & \\
\hline $25-34$ & 966 & 56.7 & 2466 & 50.8 & & 3432 & 52.3 & 475 & 39.6 & \\
\hline $35-44$ & 395 & 23.2 & 1433 & 29.5 & & 1828 & 27.9 & 257 & 21.4 & \\
\hline $45+$ & 78 & 4.6 & 405 & 8.3 & & 483 & 7.4 & 352 & 29.3 & \\
\hline South African & 835 & 49.0 & 2523 & 51.9 & 0.04 & 3358 & 51.2 & 636 & 53.0 & 0.26 \\
\hline \multicolumn{5}{|l|}{ Calendar year of study recruitment } & $<0.001$ & & & & & \multirow[t]{4}{*}{$<0.001$} \\
\hline$\leq 1992$ & 426 & 25.0 & 1816 & 37.4 & & 2242 & 34.2 & 450 & 37.4 & \\
\hline 1993 & 338 & 19.9 & 3043 & 62.6 & & 3381 & 51.5 & 634 & 52.8 & \\
\hline $1994+$ & 939 & 55.1 & 0 & 0 & & 939 & 14.3 & 118 & 9.8 & \\
\hline Still employed in 2002 & 444 & 26.1 & 1855 & 38.2 & $<0.001$ & 2299 & 35.0 & 110 & 9.2 & $<0.001$ \\
\hline HIV-positive & \multicolumn{2}{|l|}{-} & \multicolumn{2}{|l|}{-} & & 1703 & 26.0 & \multirow{2}{*}{\multicolumn{2}{|c|}{$\begin{array}{l}247 \quad 20.6 \\
4.8(2.5 \text { to } 5.9)\end{array}$}} & $<0.001$ \\
\hline Median (IQR) study follow-up, years & \multicolumn{2}{|c|}{$4.9(2.5$ to 7.0$)$} & \multicolumn{2}{|c|}{$6.6(4.0$ to 9.4$)$} & $<0.001$ & \multicolumn{2}{|c|}{$6.0(3.3$ to 9.3$)$} & & & \multirow[t]{2}{*}{$<0.001$} \\
\hline $\begin{array}{l}\text { Median (IOR) follow-up, years } \\
\text { (primary analysis) }\end{array}$ & \multicolumn{2}{|c|}{$3.4(1.9$ to 5.6$)$} & \multicolumn{2}{|c|}{$5.4(3.2$ to 9.3$)$} & $<0.001$ & \multicolumn{2}{|c|}{$5.0(2.5$ to 8.7$)$} & & & \\
\hline
\end{tabular}

${ }^{*} p$ Value from test comparing HIV-positive and HIV-negative miners.

tp Value from test comparing those included and excluded from the analysis.

work in the final year of life had decreased to 224, with the time off due to medical reasons increasing to 80 days. Table 3 also shows that the $167 \mathrm{HIV}$-negative miners who died from natural causes spent $27.8 \%$ of time off work in the final year of life (264 days), 13.2\% (48 days) of which were for medical reasons. After adjustment for age and calendar period, the overall time off in the final year was higher in the $361 \mathrm{HIV}$-positive miners who died (OR 1.70; 1.35 to 2.15) and this increase was primarily due to medical absence (RRR 2.05; 1.42 to 2.97).

Figure 1 shows that, in HIV-positive miners, after an initial increase in time off work, compared with HIV-negative men, the overall proportion remained broadly stable for a number of years, then rose in the 2 years prior to death. Medical leave follows a similar pattern, but with a gradual increase over the 8-year period, and dramatic rise in the final year.

\section{Effect of HIV infection by time since seroconversion, adjusting} for age and calendar period

In the adjusted analysis (table 4), overall absence from work, relative to HIV-negative miners, increased in the 2 years after seroconversion (adjusted OR 1.40, 95\% CI 1.36 to 1.45) and remained at this increased odds for some years, rising to an OR of 3.27 (2.95 to 3.63) in the year before death. Medical absence shows the strongest link to HIV infection and time since seroconversion, with adjusted RRR relative to being at work and relative to HIV-negative miners of 2.66 (2.45 to 2.90) for the first 2 years after seroconversion, increasing gradually over time and reaching 13.6 (11.8 to 15.6) in the year prior to death. Although the crude proportion of time off due to unauthorised absence decreased with time since seroconversion, this was confounded by calendar period, and after adjustment, unauthorised absence

Table 2 Descriptive analysis of the proportion of time at and off work

\begin{tabular}{|c|c|c|c|c|c|}
\hline \multirow[b]{2}{*}{ Factor } & \multirow[b]{2}{*}{ At work (\%) } & \multicolumn{3}{|c|}{ Off work (\%) } & \multirow[b]{2}{*}{ Total, (person-years } \\
\hline & & Medical & Leave & Unauthorised absence & \\
\hline Total & 83.0 & 2.7 & 13.1 & 1.3 & 34424 \\
\hline \multicolumn{6}{|l|}{ HIV status } \\
\hline Positive & 79.3 & 5.5 & 13.4 & 1.9 & 6643 \\
\hline Negative & 83.9 & 2.1 & 13.0 & 1.1 & 27781 \\
\hline \multicolumn{6}{|l|}{ Calendar period } \\
\hline $1992-1995$ & 84.3 & 1.7 & 12.3 & 1.7 & 7080 \\
\hline $1995-1996$ & 81.9 & 2.5 & 14.0 & 1.7 & 8764 \\
\hline 1997-1998 & 82.1 & 3.6 & 13.2 & 1.1 & 8206 \\
\hline 1999-2000 & 83.7 & 2.8 & 12.7 & 0.8 & 6019 \\
\hline $2001-2002$ & 83.7 & 3.1 & 12.8 & 0.5 & 4354 \\
\hline \multicolumn{6}{|l|}{ Age (years) } \\
\hline$<30$ & 82.9 & 2.6 & 12.6 & 2.0 & 5237 \\
\hline $30-39$ & 83.2 & 2.6 & 13.0 & 1.2 & 17539 \\
\hline $40+$ & 82.7 & 2.9 & 13.4 & 1.0 & 11648 \\
\hline \multicolumn{6}{|c|}{ No of years since HIV seroconversion } \\
\hline$<2$ & 78.5 & 4.8 & 14.2 & 2.6 & 1927 \\
\hline 2 to $<4$ & 79.5 & 5.2 & 13.3 & 2.0 & 1972 \\
\hline 4 to $<6$ & 79.6 & 6.0 & 12.9 & 1.5 & 1532 \\
\hline 6 to $<8$ & 79.9 & 5.9 & 13.0 & 1.2 & 857 \\
\hline $8+$ & 79.3 & 7.1 & 12.6 & 1.0 & 355 \\
\hline
\end{tabular}


Table 3 Work attendance by HIV status and time period before death for 361 HIV-positive and 167 HIV-negative miners who died from natural causes

\begin{tabular}{|c|c|c|c|c|c|c|c|}
\hline Work status & \multicolumn{3}{|c|}{$\begin{array}{l}\text { HIV-positive } \\
\text { (\%) time, by years before death }\end{array}$} & \multicolumn{3}{|c|}{$\begin{array}{l}\text { HIV-negative } \\
\text { (\%) time, by years before death }\end{array}$} & $\begin{array}{l}\text { Final year: adjusted } \neq \text { OR or RRR }(95 \% \mathrm{CI}) \\
\text { HIV-positive versus HIV-negative }\end{array}$ \\
\hline At work & 78.4 & 76.3 & 61.2 & 81.9 & 76.2 & 72.2 & \\
\hline Medical & 5.7 & 8.6 & 21.8 & 3.6 & 7.5 & 13.2 & $2.05 \dagger(1.42$ to 2.97$)$ \\
\hline Leave & 13.4 & 13.2 & 14.4 & 12.9 & 14.7 & 12.8 & $1.34 \dagger(1.06$ to 1.68$)$ \\
\hline Unauthorised & 2.6 & 1.9 & 2.6 & 1.6 & 1.6 & 1.9 & $1.67 \dagger(1.19$ to 2.34$)$ \\
\hline
\end{tabular}

*OR for work absence, HIV-positive versus HIV-negative.

†RR ratio for type of work absence relative to being at work, HIV-positive versus HIV-negative.

$\ddagger$ Adjusted for age and calendar period.

was greater in HIV-positive men, with an RRR of 1.95 (1.84 to 2.06 ) in the first 2 years, increasing to 3.75 (3.15 to 4.46) in the final year before death. In contrast, authorised leave was only modestly elevated in HIV-positive miners and demonstrated little association with time since seroconversion; the greatest adjusted RRR for authorised leave was 1.51 (1.31 to 1.73) seen in the year prior to death.

Repeating the analysis based on commencing follow-up time at study entry rather than at the start of records of time off work increased the time spent at work, so that the percentage of time off work for medical reasons, leave and unauthorised absence was reduced to $4.3 \%, 10.6 \%$ and $1.5 \%$ in HIV-positive miners and $1.8 \%, 11.5 \%$ and $1.0 \%$ in HIV-negative miners respectively. The associations between HIV infection and work absence were broadly unchanged, though with some reduction in the apparent effect of HIV infection in the first years following seroconversion. The adjusted OR (95\% CI) for time off work relative to HIV-negative miners was 1.00 (0.95 to 1.04) in the first 2 years following seroconversion, increasing to 1.43 (1.32 to 1.56$)$ at 8 years or more and reaching 3.43 (3.09 to 3.81) in the year before death. Considering time off work for medical reasons the adjusted RRR ( $95 \% \mathrm{CI})$ relative to being at work and to HIV-negative miners was 1.95 (1.79 to 2.13 ) in the 2 years following seroconversion increasing to 3.49 (2.88 to 4.24$)$ at 8 years or more and reaching 14.2 (12.3 to 16.3) in the year before death.

\section{Time off work in HIV-positive men before and after seroconversion}

In this sensitivity analysis of 819 miners, overall time off work increased after seroconversion from $17.6 \%$ to $19.8 \%$ in the first

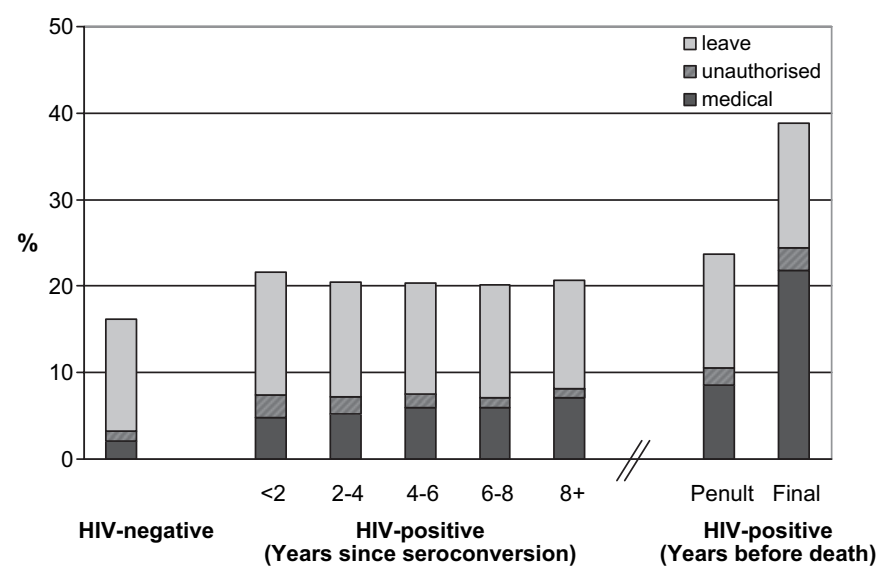

Figure 1 Proportion of time off work, by reason, HIV status, years since seroconversion and years before death.
2 years (OR 1.16; 1.11 to 1.21 ). Medical absence increased from $2.4 \%$ preseroconversion to $4.1 \%$ in the first 2 years (RRR 1.80; 1.59 to 2.03$)$.

\section{DISCUSSION}

In this large study that followed a cohort of seroconverters and an HIV-negative control group for up to 10 years, we found that HIV-positive miners spent more time off work than HIV-negative miners, mainly due to greater medical absence. The increased time off work was apparent soon after seroconversion, rose with duration of HIV infection (albeit modestly in the first 8 years) and reached very high levels in the later stages of disease, with men being off work nearly $40 \%$ of the time in the year prior to death. HIV/AIDS has a profound impact on both the supply of labour and demand for medical care.

The study strengths include a large sample size, an HIVnegative comparison group and a long follow-up. Linking to mortality data and analysing time off in the years prior to death also provide maximum estimates for the period when individuals are likely to have AIDS. Unlike self-reported indicators of health, with the potential for recall and response bias, time off data are routinely collected. Furthermore, in contrast to settings where sickness absence may be taken for reasons other than illness, in the mines sick leave is granted only following a visit to the health services.

The study may be limited by the incompleteness of the data as represented in gaps in the dataset or a total absence of time off records, although this was not directly linked to HIV status. Repeating the analysis based on commencing follow-up time at study entry rather than at the start of records of time off work, we found more time spent at work for both HIV-positive and HIV-negative miners, as expected, but broadly similar associations between work absence and HIV infection. Although we were able to exclude those HIV-negative miners who we knew had become HIV-positive, the HIV-negative comparison group is likely to include undetected seroconverters, thus diluting the effects we found. We recognise that the cohort includes a different follow-up over calendar period and age for different miners, but we control for these factors in the analysis, and present the effect of HIV infection by time since seroconversion.

It is often the healthiest men who are recruited, and those remaining in employment are fit enough to endure strenuous physical labour; the 'healthy worker effect.' The absolute number of days off as a proxy for morbidity is therefore probably an underestimate, yet relative risks may be little affected. HIV-positive workers may be inherent risk-takers before and after infection. In our sensitivity analysis, we were able to remove any such bias by using the preseroconversion period as the baseline. The effects on time off work overall and medical 
Table 4 Effect of HIV on time off work, adjusted for calendar period and age

\begin{tabular}{|c|c|c|c|c|}
\hline Factor & Overall absence from work, $\mathrm{OR}$ & Medical, RRR $\neq$ & Leave, RRR $\neq$ & Unauthorised, RRR $\ddagger$ \\
\hline $\begin{array}{l}\text { HIV status and years since } \\
\text { seroconversion* }\end{array}$ & $\mathrm{p}<0.001$ & $\mathrm{p}<0.001$ & $\mathrm{p}<0.001$ & $\mathrm{p}<0.001$ \\
\hline Negative & $1-$ & $1-$ & $1-$ & $1-$ \\
\hline Positive: $<2$ & $1.40(1.36$ to 1.45$)$ & 2.66 (2.45 to 2.90$)$ & $1.16(1.13$ to 1.19$)$ & 1.95 (1.84 to 2.06$)$ \\
\hline Positive: 2 to $<4$ & $1.31(1.27$ to 1.35$)$ & 2.57 (2.35 to 2.81 ) & $1.06(1.03$ to 1.10$)$ & $1.82(1.70$ to 1.93$)$ \\
\hline Positive: 4 to $<6$ & $1.35(1.29$ to 1.40$)$ & 2.81 (2.53 to 3.11 ) & 1.06 (1.02 to 1.09$)$ & 1.96 (1.80 to 2.14$)$ \\
\hline HIV status and years before death $\dagger$ & $\mathrm{p}<0.001$ & $\mathrm{p}<0.001$ & $\mathrm{p}<0.001$ & $\mathrm{p}<0.001$ \\
\hline Negative & $1-$ & $1-$ & $1-$ & $1-$ \\
\hline Positive: $>2$ years before death & $1.41(1.34$ to 1.49$)$ & 3.05 (2.62 to 3.56$)$ & 1.09 (1.05 to 1.14$)$ & 2.10 (1.90 to 2.32$)$ \\
\hline Positive: penultimate year & $1.59(1.45$ to 1.73$)$ & 4.31 (3.62 to 5.13 ) & $1.10(1.01$ to 1.20$)$ & 2.03 (1.75 to 2.35$)$ \\
\hline Positive: final year & 3.27 (2.95 to 3.63$)$ & 13.6 (11.8 to 15.6$)$ & 1.51 (1.31 to 1.73 ) & 3.75 (3.15 to 4.46$)$ \\
\hline
\end{tabular}

*Adjusted OR and RR ratio (RRR) with $95 \% \mathrm{Cl}$ are from a regression model including calendar time, age and HIV status/years since seroconversion.

†Adjusted OR and RRR with $95 \% \mathrm{Cl}$ are from a regression model including calendar time, age and HIV status/years before death. All data from HIV-negative miners are used together with data from those HIV-positive miners who died during follow-up from natural causes.

$\ddagger R R$ ratio values with $95 \% \mathrm{Cl}$ are given relative to being at work.

absence were slightly reduced but remained significant in the first 2 years following seroconversion.

By following a seroconverter cohort prospectively, as well as looking retrospectively at the period before death, our study quantifies all-cause morbidity over the entire spectrum of HIV disease. We have previously shown that the effect of HIV on tuberculosis incidence ${ }^{17}$ and mortality ${ }^{16}$ occurs soon after infection. Similarly, within the first 2 years, HIV-positive men are likely to have a larger proportion of time off work overall and for medical reasons, suggesting early effects on overall morbidity. In the terminal phase, comparing men who died from natural causes, time off work was greater in the HIV-positive men than in HIV-negative men, suggesting a more chronic decline. In this setting, infectious causes of death predominated ( $88 \%$ of natural deaths in HIV-positive and $41 \%$ in HIV-negative men), with tuberculosis the leading cause of death in both groups. ${ }^{19}$

The implications of HIV on industry and the workforce are multifaceted. With disease progression, and in the absence of ART, HIV-positive workers are likely to have increasing time off work, declining productivity and rising mortality. This study does not provide data on productivity in the workplace, which is difficult to measure. Even if at work, HIV may be impacting on productivity or on workplace organisation (so-called presenteeism), such as the delegation of less strenuous tasks ${ }^{13}$ or fellow workers carrying the workload for those who are sick. Financial incentives set a high threshold for taking time off. A study of 54 Kenyan tea estate workers who died or took medical retirement found that HIV-positive workers plucked less tea per day (a measure of work output), took more sick and annual leave, and earned $18 \%$ less in their final year. ${ }^{10}$ The results are an underestimate, since the HIV status of the comparison group was unknown, and helpers were allowed to assist sick workers. The impact on service delivery capacity may be more marked in the public sector: the $45 \mathrm{HIV}$-positive employees of the Zambian Wildlife Authority (ZAWA) who died had worked 68\% less (63 vs 197 days) in their last year of service compared with in-service employees. $^{20}$ In the Swiss HIV Cohort Study, ${ }^{14}$ there were substantial productivity losses, measured as ability to work and monthly wages, and these were more likely in individuals with worse clinical prognostic factors (older, longer duration of HIV, lower CD4 count and a history of AIDS). Ability to work was also dependent on other socio-economic factors that impact on earning potential, such as gender, educational level and stable partnerships. ${ }^{14}$ Similarly, we found that individuals who were older or had longer durations of HIV infection had more time off work.

HIV/AIDS results in higher worker turnover, loss of a skilled labour force and an increased need for training. There is also a loss of human capital: the experience and collectively acquired knowledge of workers who die or leave employment. ${ }^{6}$ The effect of supply shortages in the healthcare, public and educational sectors, resulting from HIV infection in employees, is likely to have even larger societal impacts. As early as 1990, the National Railways of Zimbabwe reported operational problems due to absenteeism of $>15 \% .^{9}$ Furthermore, there is likely to be reduced staff morale ${ }^{21}$ and changes in the status of men who had previously been household breadwinners. ${ }^{22}$ HIV can be seen to have even greater impacts on individuals, families and communities when one considers not only time off while in employment, but more broadly the years of productive life lost, which includes time after leaving the mine for any reason, including death. This theoretical time lost assumes that HIV-positive men would have remained in healthy employment until retirement age, rather than dying or becoming ill earlier, and recognises that those who leave may be unlikely to gain employment elsewhere.

This study shows that HIV-positive individuals will require increasing and substantial medical care, with implications for the provision of health services both in the workplace and in home areas. Relative to HIV-negative miners, time off for medical reasons in those who are HIV-positive increased soon after infection (RRR 2.7 in the first 2 years) and reached $23 \%$ of total time in the last year of life (RRR 13.6). A study in another gold mine found a significant difference in all-cause hospitalisation rates between HIV-infected and non-infected employees, 2 years prior to death. ${ }^{4}$ Yet, hospitalisation only accounts for a small proportion of days lost for medical reasons. ${ }^{12}$ HIV has had a dramatic effect on the burden of tuberculosis, with both a direct impact on incidence in HIV-positive individuals and a secondary indirect increase in the HIV-negative population, ${ }^{23}$ which would further impact on demand for health services. HIV also increases the risk of work-related injuries with implications for medical care and compensation. ${ }^{18}$

This study, although conducted prior to the introduction of ART, has important implications that are relevant currently, and for the future. Despite the rollout of ART, the impact of HIV is likely to continue, albeit on a smaller scale. Preliminary findings from 59 HIV-positive Kenyan agricultural workers suggest that, despite higher productivity in the first year after starting ART, 
absenteeism and productivity may not return to HIV-negative levels. ${ }^{11}$ A recent study in three South African clinics found that, compared with pre-ART patients, those on ART reported less functional impairment and better work performance; however, there remained a high prevalence of some impairment, symptoms and absenteeism. ${ }^{24}$ Follow-up of similar cohorts to the one in this study, including the period when ART is readily available, will provide insights into the impact of treatment on time off work and whether this is sustained over time.

Many HIV-infected individuals present to health services with advanced immunosuppression, and this is higher in men. ${ }^{25}$ Occupational health services are an important setting in which to identify HIV-infected men early. Occupational settings may also have the infrastructure and expertise to provide HIVprevention campaigns, increased uptake of testing, wellness clinics and early ART. In addition to the impact on industry and the workforce, our findings suggest that HIV/AIDS will result in a greater need for healthcare, with implications for health services and resource allocation. Since the effects of HIV on morbidity and mortality start soon after seroconversion, at a time prior to the recommendation for commencement of ART, even with an ART programme there will be an increased need for healthcare overall, in addition to the implications of providing lifelong ART.

Evaluations of interventions need to consider the effect on delaying or averting HIV-related costs. A key element of any workplace intervention would be a multisectoral approach including workers, the private sector, communities and government. ${ }^{26}$ Interventions need to consider a broader 'combination prevention' approach that integrates behavioural, biomedical and structural HIV-prevention strategies with HIV treatment. ${ }^{27}$ This study provides empirical data that can be used as a baseline to estimate the cost-effectiveness of interventions and to support the International Labour Organisation statement that "the direct and indirect costs of inaction are far greater than the costs of treatment. 6

Acknowledgements We would like to thank the staff of the Human Resources and Medical Departments at Gold Fields Ltd for help with accessing current and archived datasets, and for sharing insights into the structure of the workforce and allocation of leave.

Funding Colt Foundation UK.

Competing interests There are no competing interests. Mine management did not have access to the study dataset, and played no role in the study design or interpretation of results.

Ethics approval Ethics approval was provided by the Ethics Committees at the University of the Witwatersrand, South Africa and LSHTM, UK.

Provenance and peer review Not commissioned; externally peer reviewed.

\section{REFERENCES}

1. Evian C, Fox M, MacLeod W, et al. Prevalence of HIV in workforces in southern Africa, 2000-2001. S Afr Med J 2004:94:125-30.
2. Petschel EG, Lowe RE, Murray J. The Course of HIV Infection in a Southern African Cohort. Durban, South Africa, 1993. 12th Epidemiological Conference.

3. Campbell CM, Williams BG. Managing disease on the goldmines. 'Work-related' and 'non-work-related' diseases. S Afr Med J 1998:88:789-95.

4. Corbett EL, Churchyard GJ, Charalambos S, et al. Morbidity and mortality in South African gold miners: impact of untreated disease due to human immunodeficiency virus. Clin Infect Dis 2002:34:1251-8.

5. Arndt C, Lewis JD. The macro implications of HIV/AIDS in South Africa: a preliminary assessment. S Afr J Econ 2000:68:380-92.

6. ILO Programme on HIV/AIDS and the world of work. HIVIAIDS and Work: Global Estimates, Impact and Response. Switzerland: International Labour Organisation, 2004

7. Quattek K, Fourie T. The Economic Impact of AIDS in South Africa: A Dark Cloud on the Horizon. London: ING Barings, 2000.

8. Marseille E, Saba J, Muyingo S, et al. The costs and benefits of private sector provision of treatment to HIV-infected employees in Kampala, Uganda. AIDS 2006:20:907-14

9. Bollinger L, Stover J, Kerkhoven R, et al. The economic impact of AIDS in Zimbabwe. The Futures Group International in collaboration with: Research Triangle Institute (RTI) and The Centre for Development and Population Acivities (CEDPA) 1999.

10. Fox MP, Rosen S, MacLeod WB, et al. The impact of HIV/AIDS on labour productivity in Kenya. Trop Med Int Health 2004:9:318-24.

11. Larson BA, Fox MP, Rosen S, et al. Early effects of antiretroviral therapy on work performance: preliminary results from a cohort study of Kenyan agricultural workers. AIDS 2008:22:421-5.

12. Morris CN, Burdge DR, Cheevers EJ. Economic Impact of HIV Infection in a Cohort of Male Sugar Mill Workers in South Africa. S Afr J Econ 2000;68:413-19.

13. Rosen S, Feeley F, Connelly P, et al. The private sector and HIV/AIDS in Africa: taking stock of 6 years of applied research. AIDS 2007;21(Suppl 3):S41-51.

14. Sendi P, Schellenberg F, Ungsedhapand C, et al. Productivity costs and determinants of productivity in HIV-infected patients. Clin Ther 2004;26:791-800.

15. Concerted Action on SeroConversion to AIDS and Death in Europe. Time from HIV-1 seroconversion to AIDS and death before widespread use of highly-active antiretroviral therapy: a collaborative re-analysis. Collaborative Group on AIDS Incubation and HIV Survival including the CASCADE EU Concerted Action. Lancet 2000;355:1131-7.

16. Glynn JR, Sonnenberg P, Nelson G, et al. Survival from HIV-1 seroconversion in Southern Africa: a retrospective cohort study in nearly 2000 gold-miners over 10 years of follow-up. AIDS 2007;21:625-32.

17. Sonnenberg $\mathbf{P}$, Glynn JR, Fielding $\mathrm{K}$, et al. How soon after infection with HIV does the risk of tuberculosis start to increase? A retrospective cohort study in South African gold miners. J Infect Dis 2005;191:150-8.

18. Murray J, Sonnenberg P, Nelson G, et al. Effect of HIV on work-related injury rates in South African gold miners. AIDS 2005:19:2019-24.

19. Murray J, Sonnenberg P, Nelson G, et al. Cause of death and presence of respiratory disease at autopsy in an HIV-1 seroconversion cohort of southern African gold miners. AIDS 2007;21(Suppl 6):S97-104

20. Rosen S, Hamazakaza P, Feeley F, et al. The impact of AIDS on government service delivery: the case of the Zambia Wildlife Authority. AIDS 2007:21(Suppl 3):S53-9.

21. Phaswana-Mafuya N, Peltzer K. Perceived HIV/AIDS impact among staff in tertiary institutions in the Eastern Cape, South Africa. SAHARA J 2005;2:277-85.

22. Tersbol B. 'I just ended up here, no job and no health'-men's outlook on life in the context of economic hardship and HIV/AIDS in Namibia. SAHARA J 2006;3:403-16.

23. Sonnenberg $\mathbf{P}$, Glynn JR, Fielding $\mathrm{K}$, et al. HIV and pulmonary tuberculosis: the impact goes beyond those infected with HIV. AIDS 2004;18:657-62.

24. Rosen S, Kethapile M, Sanne I, et al. Differences in normal activities, job performance and symptom prevalence between patients not yet on antiretroviral therapy and patients initiating therapy in South Africa. AIDS 2008;22(Suppl 1):S131-9

25. Cornell M, Myer L, Kaplan R, et al. The impact of gender and income on survival and retention in a South African antiretroviral therapy programme. Trop Med Int Health 2009:14:722-31.

26. Oppenheimer J. Business and AIDS in South Africa. AIDS 2007;21(Suppl 3):S11-12

27. Coates TJ, Richter L, Caceres $\mathrm{C}$. Behavioural strategies to reduce HIV transmission: how to make them work better. Lancet 2008:372:669-84. 